\title{
Desafíos de la Propiedad Intelectual frente al rol del influencer en la industria de la moda.
}

\author{
Intellectual property challenges in the \\ influencer's role in fashion industry
}

\author{
Julia Cañas Coloma* \\ Investigadora Jurídica Independiente \\ Infomación del Artículo \\ Original - Ruptura, 2020 \\ Artículo recibido / Received: 4 de junio, 2020 \\ Artículo aceptado / Accepted: 28 de julio, 2020
}

\section{Citación}

Cañas, J. (2020). Desafíos de la propiedad intelectual frente al rol del influencer en la industria de la moda. Revista Ruptura de la Asociación Escuela de Derecho PUCE. Edición 2020, p (563-575).

DOI: $10.26807 /$ rr.vi02.61

Resumen: El presente artículo tiene como objetivo plasmar los desafíos de la propiedad intelectual en la actualidad, enfocado principalmente en el marketing realizado por influencers que cada día toman más cabida en la industria de la moda y es considerado como una alternativa al pensamiento de publicidad clásica. Desde una perspectiva jurídica, se consideran los efectos y las posibles alternativas que debería tomar el Ecuador para regular este nuevo marketing, que en nuestro país, está tomando gran importancia.

Palabras clave: Propiedad intelectual, influencer, marketing, publicidad, marca, derechos de autor.

* Estudiante de cuarto semestre de la carrera de Derecho de la Facultad de Jurisprudencia de la Pontificia Universidad Católica del Ecuador. 


\begin{abstract}
This article aims to capture intellectual property challenges today, mainly focused on the influencer marketing that is increasingly taking place in the fashion industry and it is considered as an alternative to classic advertising thinking. From a legal perspective, the effects and possible alternatives that Ecuador should evaluate to regulate this new marketing are considered, which in our country, is taking on great importance.
\end{abstract}

Key words: Intellectual property, influencer, marketing, advertising, brand, copyright.

\title{
Introducción
}

Con el avance de la tecnología, la evolución de las redes sociales y el incremento de seguidores en las mismas, han surgido sin lugar a dudas, nuevos retos para la propiedad intelectual a nivel mundial. En la actualidad la promoción de las grandes marcas se realizan a través de las redes sociales con la participación activa y estelar de los denominados influencers, cuya actividad no está legalmente regulada en el país, misma que podría acarrear conflictos respecto del uso de la marca y los derechos de autor.

El presente artículo, busca analizar este tema, para lo cual se determinarán ciertos conceptos como, ¿quién es un influencer?, y i̇cuál es su rol en la promoción de las marcas? También se analizará la importancia del marketing influencer en contraposición con los derechos de propiedad intelectual que pueden verse afectados; sus efectos en la economía y el análisis respecto de la falta de regulación de esta actividad en nuestro país.

Las fuentes de consulta bibliográfica utilizadas para la elaboración de este artículo, son principalmente recursos obtenidos de internet, así como las principales cuentas de redes sociales de quienes promocionan marcas de conocidas empresas multinacionales de la moda. Adicionalmente, podrán contrastarse las regulaciones legales vigentes de esta materia en otros países, con el fin de responder a las incógnitas y dilucidar si esta regulación existe en nuestro país y si es realmente necesaria. 


\section{El influencer}

En la actualidad, el término "influencer" hace referencia a jóvenes o figuras mediáticas que tienen, como la misma palabra lo menciona, cierto tipo de "influencia". Especialmente, en personas cuya edad comprende entre los 16 y 35 años, recurrentes usuarios de redes sociales y público objetivo de marcas e industrias que los consideran sus compradores principales.

En otras palabras, y de acuerdo a lo explicado por, Annalucia Fasson, se puede entender que:

Los influencers son aquellas personas que tienen un blog, página web y/o canal de YouTube. En estas plataformas digitales cuentan sus experiencias sobre el uso de un producto o servicio en particular e influyen así en que sus seguidores se animen a comprar, viajar, comer o experimentar alguna actividad. (Fasson, 2017)

Estas nuevas figuras que lideran en seguidores en plataformas como Instagram surgen con el nuevo milenio, con el auge de las redes sociales y los blogs. Gracias a su acogida en la generación millenial y centenial, se han convertido en el medio predilecto de todo tipo de marcas para anunciar sus productos o servicios, con el fin de aumentar sus ventas. La accesibilidad al internet de forma libre y sin restricciones, han sido la clave para que el marketing influencer pueda funcionar, pues en la actualidad, se considera que la televisión y la radio ya no son medios eficaces para publicitar dado que, ahora las redes sociales permiten difundir ideas de forma inmediata, con una respuesta rápida por parte del consumidor, y con un mayor dinamismo, lo cual facilita a las marcas comerciales para conocer de primera mano qué influencers y qué contenidos funcionan mejor para publicitar un producto o servicio.

De esta forma, en los últimos años han surgido nuevas aplicaciones para transmitir a la audiencia cierta opinión, fotografía o compaña sobre cualquier índole. Este el caso de historias en Instagram (instastories), publicaciones en Facebook, IG-TV, transmisiones en directo y vídeos de YouTube. 
Hoy en día, los influencers tienen una gran repercusión e importancia en el mercado, por un hecho simple : poseen habilidades comunicativas que les permiten transmitir a un público, que confía en ellos, lo que las marcas quieren vender. Los consumidores tienen acceso a información real, de personas a las que admiran. Esta confianza proviene de la experiencia que cada influencer transmite, pues comentan en tiempo real su opinión y experiencia sobre el producto. Lo antes mencionado es un punto clave, pues se demuestra la superación a medios tradicionales, donde si bien se probaba el producto, esto no era con tal cercanía y fuera de libretos llamativos para el consumidor.

Gracias a estos nuevos publicistas, las empresas pueden llegar de forma más fácil y rápida a una masa social que es aquella que sigue al influencer en cuestión. Esto puede ayudar a mejorar la imagen de la marca y aumentar su visibilidad en un menor tiempo y con un mayor alcance. Además, con un manejo estratégico del marketing influencer, pueden lanzarse nuevos productos, marcas o incluso difundir eventos.

Entre los influencers más destacados está Cristiano Ronaldo, jugador estrella del equipo de fútbol italiano Juventus. Quien cuenta con más de 222 millones de seguidores en Instagram. Estas cifras han llamado la atención de innumerables marcas como Nike, que ha promocionado sus productos a través del jugador. Lo cual genera una ilusión en el consumidor de adquirir cierta indumentaria que un grande del fútbol "aprueba". En Ecuador, se puede tomar como referencia a María Teresa Guerrero, ex presentadora de televisión. Misma que ahora promociona ropa para practicar atletismo y otros deportes en sus redes sociales. Actualmente, cuenta con más de 1.3 millones de seguidores en su cuenta de Instagram ${ }^{1}$

Por estas y muchas razones, la industria de la moda ${ }^{2}$ tiene en la mira a los influencers, pues estos se han enfocado en promover

1 Instagram de María Teresa Guerrero: https://www.instagram.com/flacaguerrerog/

2 Cuando se habla de "industria de la moda" nos referimos a un sector productivo o a una actividad económica y técnica que consiste en la realización de prendas de 
contenido visual que permite publicitar ropa y zapatos; especialmente a través de la plataforma de Instagram, que en cuestión de años revolucionó esta industria, y lo sigue haciendo. Incluso recientemente, se implementó la opción de hacer compras de forma directa desde la mencionada plataforma mediante redirección a los links de las páginas, o con las etiquetas de precios que pueden ser colocadas en las fotos donde se muestran los productos

\section{El marketing influencer y la propiedad intelectual}

Una marca, es un signo que se utiliza para distinguir productos o servicios. Permite al consumidor su identificación en el mercado y puede entenderse adicionalmente (Laver, 2005) como una reputación en el mercado que tiene una identidad (el origen de la marca), traducido en una imagen (la percepción de los clientes) que confiere un valor competitivo a las ventas adicionales, o precios altos, o ambos.

La industria de la moda, ser entendida como un sector productivo o actividad económica y técnica que consiste en la realización de prendas de vestir y complementos basados en gustos y usos de un mercado específico. Esta ha evolucionado a la par con el avance del desarrollo tecnológico de las redes sociales. Ahora, respecto a la percepción que actualmente se tiene sobre la publicidad de las marcas, grandes empresas han decidido hacer uso de la idea de los influencers para promocionar sus bienes y/o servicios, ya sea como "fashion bloggers", que son aquellos que modelarán una colección de la marca para una determinada campaña, o simplemente contratándolos para que sean una referencia de la marca y la recomienden.

Sin embargo, surge una cuestión interesante para el mundo jurídico, pues se presenta un problema de derechos de propiedad intelectual cuando los influencers generan este contenido para grandes industrias de la moda. Por una parte, hacen uso de una

vestir y complementos basados en gustos y usos, que se utilizan durante un periodo de tiempo determinado y que marcarán tendencia según la duración de este. 
marca registrada, debidamente protegida y posicionada en el mercado, es decir, con cierta reputación. Esto plantea la duda de quién efectivamente posee los derechos de autor cuando nos referimos al contenido per se, que ha sido creado en una campaña de publicidad con un influencer, aspecto que en nuestro país no ha sido regulado desde el ámbito legal.

En el ámbito jurídico, la expresión "derecho de autor" se utiliza para describir los derechos de los creadores sobre sus obras literarias y artísticas. Las obras que se enmarcan en la protección por derecho de autor van desde los libros, la música, la pintura, la escultura y las películas, hasta los programas informáticos, las bases de datos, los anuncios publicitarios, los mapas y los dibujos técnicos y en este caso, aspectos de la moda como son diseños de ropa y zapatos.

Cuando se habla de una campaña influencer, generalmente son los influencers los que generan la idea y el contenido de la campaña con su propio tiempo, intelecto creativo, espacio y recursos. Un trabajo que va desde buscar locaciones adecuadas, edición de fotos y/o vídeos de la campaña, y diseño de los diferentes elementos o características que comprenderán la imagen posteriormente publicada. Por lo que, si entre el propietario de la marca y quien ejecutará la campaña -entiéndase todo el proceso creativo antes mencionado- no se ha establecido previamente un contrato que afirme lo contrario, la propiedad intelectual del contenido producido para la campaña va a pertenecer al influencer. Esto significa que la compañía propietaria de la marca no podrá recrear, reproducir o reusar el contenido de la campaña sin el consentimiento expreso del influencer.

En este escenario, para un mejor manejo de los derechos de propiedad intelectual, derivados de una campaña publicitaria con marketing influencer, es primordial que las empresas regulen este tipo de convenios a modo de contratación, estableciendo términos y condiciones para determinar a quién le pertenece la propiedad intelectual de lo producido. Además, podría establecerse, por ejemplo, el derecho de la empresa propietaria de la marca a usar exclusivamente el contenido creado por un periodo de tiempo específico, o permitir que el influencer sea el dueño del contenido, 
con la salvedad de que la empresa propietaria de la marca posea el derecho a usarlo en campañas o anuncios futuros.

El hecho de que las empresas propietarias de las marcas promocionadas, sean las que se atribuyan los derechos de autor de la campaña publicitaria, les permite establecer un control sobre el contenido que es publicado por el influencer, con el fin de proteger y cuidar el prestigio de su marca, limitando al creador de contenido a lo que la empresa desee mostrar, que evita así la dilución o debilitamiento de la marca. Alineando esto además, a la misión de la campaña y sin que se dañe la esencia del producto o servicio, o se difunda una mala publicidad que dañe la imagen de la marca y lo que se quiere vender.

Por ello, es necesario y de suma importancia que entre las empresas y los influencers se suscriban contratos o se plasmen acuerdos que regulen adecuadamente todos estos aspectos, con el fin de garantizar la seguridad jurídica para las dos partes; por un lado, la protección del prestigio de una determinada marca, y por otra, la protección de los derechos de autor que tienen los influencers en la creación de contenidos para determinada campaña u otra actividad publicitaria.

En este sentido, según lo mencionado por Mónica Ramos Benítez, quien realiza un análisis de la legislación en Puerto Rico, en el contrato de marketing influencer debe incluirse ciertos aspectos básicos tales como:

Debe incluirse en el contrato la obligación de cumplimiento con las guías de la Comisión Federal de Comercio (FTC, por sus siglas en inglés) para la publicidad y mercadeo en redes sociales, las cuales requieren, entre otras cosas, que los influencers divulguen cuando el contenido publicado es una promoción o anuncio pagado utilizando hashtags como \#ad o \#sponsored. La inclusión de esta obligación en el contrato provee una protección para las compañías ante un posible incumplimiento por el influencer con las guías de la FTC. (Ramos Benítez, 2019)

Cualquier acuerdo entre la empresa propietaria de la marca y el influencer debe estar establecido por escrito, de esta forma se evitarán confusiones o conflictos al momento de reconocer a quién 
le pertenece la propiedad intelectual de la campaña y quién debe reclamar este tipo de derechos.

\section{Efectos del marketing influencer en la economía y la propiedad intelectual}

Es importante destacar que una de las ventajas del marketing influencer para las grandes empresas, particularmente las de la industria de la moda, es el ahorro de dinero en publicidad. En lugar de contratar a empresas dedicadas exclusivamente a la publicidad, que trabajan por altos costos, y utilizando medios de comunicación como televisión o internet, deciden otorgar el monopolio de la publicidad a los influencers, que realizan dicha acción de una forma más dinámica, moderna, y con un alcance masivo en el público objetivo que busca la empresa de la marca.

Y aunque esto puede afectar económicamente a las empresas enfocadas en la publicidad, también se debe tomar en cuenta que estas ahora deben adaptarse a los métodos modernos de difusión de publicidad. Una alternativa es mediante convenios con los influencers, incluso utilizando a estos agentes de difusión llegar más rápido a las empresas dueñas de las marcas.

Si las empresas de la industria de la moda, propietarias de marcas con renombre, deciden utilizar a los influencers para dar a conocer sus productos y llegar a un determinado sector del público consumidor, es necesario que mantengan cierto tipo de control, con el objetivo de que el producto o servicio sea usado de manera adecuada, con veracidad y transparencia, respetando la imagen e ideología empresarial o know how. El influencer debe informar siempre y de forma clara todos estos aspectos en el momento en que promocione un producto, pues el incumplimiento de lo mencionado puede provocar consecuencias, no solo para el creador de contenido que incumple su obligación, sino también para la empresa dueña de la marca. 
Para ello, y como se mencionó anteriormente, es imprescindible que exista un contrato de por medio. Al firmar este contrato, los influencers pueden proteger sus derechos, tal como afirma Annalucia Fasson:

Es recomendable que los influencers firmen un contrato con las marcas, a través de las agencias de publicidad o directamente con ellas. En el documento se debe establecer un plazo para que puedan ser utilizados como imagen en favor de una marca, producto o servicio. Cuando se den casos de incumplimiento, el contrato debe establecer su resolución, así como la posibilidad de ir al Poder Judicial o Arbitraje, dependiendo de la controversia. Sin embargo, en muchos casos, los influencers solo celebran contratos verbales, y esto puede provocar un problema cuando quieran exigir el pago convenido con las marcas porque no tienen nada escrito (Fasson, 2017).

Es primordial tomar en cuenta que cuando una persona se convierte en influencer de la industria de la moda, está utilizando su imagen personal para promover la venta de un producto determinado producto o servicio. Aquí entran en juego también los derechos de imagen, aquellos que le permiten a una persona "hacer un uso de tu propia imagen ya sea mediante la publicación o dejando que otros lo hagan y por otro lado, pueden prohibir que otros la usen sin su consentimiento" (Ramírez, 2015).

El derecho de imagen está consagrado en la Constitución de la República del Ecuador en su artículo 66 numeral 18. De esta forma, un influencer puede garantizar que nadie abuse de sus derechos de imagen, y en caso de que esto suceda, revocar el consentimiento de prestar este derecho o de limitar la cesión del mismo.

El manejo que actualmente se les da a los derechos de imagen, ha logrado que estos se conviertan de derechos patrimoniales y de comercialización. Basta tomar un ejemplo mencionado por Jane Wakefield en el que resalta:

La firma de Marketing Izea, con base en Winter Park, Florida, analizó lo que pagan las marcas por contenido patrocinado en internet y descubrió que en Instagram el precio de una foto promocionada pasó de US\$134 en 2014 a US\$1.642 en 2019. (Wakefield, 2019) 
La propiedad intelectual tiene que adaptarse a los tiempos modernos y las nuevas realidades que se presentan en aspectos como derecho de autor, derechos de imagen, derechos patrimoniales, licencias de uso y demás. Incluso se podría asumir que con el paso de los años, nuevos derechos enfocados en la propiedad intelectual deberán ser protegidos.

\section{Contraste legal del marketing influencer}

En Ecuador, dado que es una tendencia relativamente reciente, no existe ningún tipo de regulación o legislación sobre el marketing influencer, ni las limitaciones o condiciones que este debe tener. Esto expone a varios riesgos a quienes tienen interés en efectuar campañas influencers con esta nueva técnica de publicidad.

En Reino Unido, por ejemplo, la situación es diferente. Se ha establecido una regulación enfocada en que el influencer debe declarar cuándo y cuánto han desembolsado a su favor las empresas dueñas de las marcas, aclarar la relación que puede tener con una marca o negocio, así como no engañar a posibles compradores y demás. Como lo mencionan Alicia Costas y Albert Agustinoy:

El pasado 23 de enero, la Autoridad de Competencia y de los Mercados británica (CMA - Competition \& Markets Authority-) publicó una guía que los influencers deben seguir a fin de asegurarse el cumplimiento de las normas de protección de los consumidores. La CMA justifica la elaboración de dicha guía en que los consumidores deben saber cuándo un influencer ha recibido dinero, ha sido incentivado o ha recibido algún tipo de contraprestación por mostrar un producto o servicio en sus publicaciones. De no hacerlo, el influencer podría engañar a sus seguidores, quebrantando así las leyes británicas de protección a los consumidores, lo que conllevaría la oportuna sanción de la CMA y demás autoridades competentes. (Costas \& Agustinoy, 2019)

De igual forma, la CMA da consejos para cumplir con lo establecido en la norma como; dar un mensaje transparente, fácil de entender, oportuno y destacado sobre la naturaleza dela publicación; y que para acceder a tal información no haya necesidad de hacer clic 
para acceder a la información completa, independientemente del dispositivo utilizado.

La CMA sigue las líneas ya marcadas por la Advertising Standards Authority (organización de autorregulación publicitaria en el Reino Unido) que ya había elaborado una guía sobre influencers y la publicidad ilícita, dejando claro así la CMA que por el engaño al consumidor pueden resultar responsables de las correspondientes sanciones tanto el influencer, como su representante o incluso la marca indebidamente promocionada. (Costas \& Agustinoy, 2019)

En Ecuador, la regulación al marketing influencer debió existir desde hace varios años, cuando los influencers comenzaron a tomar importancia en el mercado. La regulación debe ir desde aspectos económicos, comerciales, civiles y de propiedad intelectual, incluyendo, tal como lo hace el Reino Unido, con lo referente a los derechos del consumidor.

Hasta que esta regulación no se lleve a cabo, Ecuador se encuentra en un estado de completo atraso, donde pueden existir vulneraciones de derechos y constantes ilegalidades. Aunque existirán aspectos que son de primordial atención al momento de legislar, no se debe restar importancia a la relevancia que tiene esta nueva forma de publicitar bienes y servicios en la actualidad, y cómo este puede beneficiar a nuestro país. Incluso en aspectos como la recaudación de impuestos y el fortalecimiento del mercado nacional.

\section{Conclusiones}

La industria de la moda ha radicado toda su atención en los influencers, pues estos se han enfocado en promover contenido visual que permite publicitar productos y servicios. Especialmente a través de diversas plataformas en internet, que en cuestión de años han logrado evolucionar la industria de la moda, y lo seguirán haciendo.

Sin embargo, esta nueva forma de hacer publicidad debe estar regulada por la ley, estableciendo convenios entre influencers y 
compañías dueñas de las marcas, donde se aclaren términos y condiciones por medio de contratos y esencialmente, que no se reste la importancia y relevancia que tiene la propiedad intelectual en estas formas modernas de difusión de contenido y publicidad.

Ecuador necesita establecer de forma inmediata y apropiada, leyes enfocadas a la regulación del marketing influencer, para evitar el cometimiento de ilegalidades, la vulneración de derechos fundamentales y de propiedad intelectual, para fomentar este tipo de negocios que cada día toman más cabida en el mundo contemporáneo.

\section{Referencias Bibliográficas}

Costas, A., Agustinoy. A. (2019). Nuevas reglas de transparencia para «Influencers» en Reino Unido. Cuatrecasas. Recuperado de: https://blog. cuatrecasas.com/propiedad-intelectual/nuevas-reglas-transparencia-influencers-reino-unido/

Fasson, A. (2017). To be influencers. Esan. Obtenido de https://www.esan. edu.pe/conexion/actualidad/2017/11/20/to-be-influencers/

Laver, P. (2005). Crear una nueva marca en una semana. Gestión 2000.

Martín-Sanz, L. (4 de Febrero de 2020). Cinco días: El país. Recuperado de: https://cincodias.elpais.com/cincodias/2020/02/03/legal/1580751696_816858.html

Portal of Competition Law. (26 de Abril de 2019). Portal of Competition Law. Recuperado de: https://uclp.eu/influencerzy-naruszaja-prawo-gdy-nie-oznaczaja-linkow-jako-reklamy/

Ramírez, N. (2015). The Legal Hat: Asesoría y Acompañamiento Legal. Recuperado de: https://www.thelegalhat.com/que-son-los-derechos-deimagen/ 
Ramos Benítez, M. (2019). La propiedad intelectual y el "influencer marketing”. Microjuris: Inteligencia Jurídica. Recuperado de: https://aldia. microjuris.com/2019/05/12/la-propiedad-intelectual-y-el-influencer-marketing/

Wakefield, J. (2019). Por qué los "influencers" ganan cada vez más dinero (y cuánto cuesta un anuncio en Facebook, Instagram o YouTube). BBC. Recuperado de: https://www.bbc.com/mundo/noticias-50433554 